\title{
Focusing properties of fractal zone plates: experimental implementation with a liquid-crystal display
}

\author{
Jeffrey A. Davis and Laura Ramirez \\ Department of Physics, San Diego State University, San Diego, California 92182-1233 \\ José A. Rodrigo Martín-Romo, Tatiana Alieva, and María Luisa Calvo \\ Departamento de Optica, Facultad de Ciencias Fisicas, Universidad Complutense de Madrid, 28040 Madrid, Spain
}

Received February 11, 2004

\begin{abstract}
We report the first experimental results, to our knowledge, with fractal zone plates implemented in a liquidcrystal display. Our results largely agree with theory for the axial irradiance distribution of these lenses. The dependence of the shape and size of the focus points on critical design parameters is discussed. Additional unpredicted features are also described. (C) 2004 Optical Society of America OCIS codes: $\quad 050.1940,050.1970,230.6120,080.3630,230.3720$.
\end{abstract}

Fractal structures have attracted a great deal of interest, beginning with the early work of Mandelbrot. $^{1} \quad$ Fractal objects are not only found in nature but also can be mathematically generated by considering particular properties related to the generator geometry, fractal dimension, and center of mass characterization. In optics and optical processing of information, there is increasing interest in implementing optical structures that exhibit fractal properties such as deterministic fractal gratings. ${ }^{2,3}$ Extending this analysis, new fractal zone plates (FZPs) are characterized by a fractal focusing structure along longitudinal coordinate $z$. Recently, ${ }^{4}$ diffraction from such fractal structures was examined theoretically. In that work the central point of interest was the formation of sequences of foci along the longitudinal direction of propagation. As the level of the fractal structure increases, the number of these focus points increases. However, no experimental evidence was given.

In this work we encode these structures onto programmable spatial light modulators (SLMs) and present the first experimental results, to our knowledge, to compare with the theory presented in Ref. 4. In doing so we present a new method for generating triadic Cantor FZPs. We analyze the focusing properties of these structures, e.g., the number of focal points and the intensity distribution as a function of longitudinal coordinate $z$.

As shown in Ref. 4, the axial irradiance distribution for a Cantor set-modulated FZP is given by

$$
\begin{aligned}
& I_{\mathrm{FZP}}(u, N, S) \\
& \quad=4 \sin ^{2}\left[\frac{\pi u}{(2 N-1)^{S}}\right] \prod_{i=1}^{S} \frac{\sin ^{2}\left[\frac{2 \pi N u}{(2 N-1)^{i}}\right]}{\sin ^{2}\left[\frac{2 \pi u}{(2 N-1)^{i}}\right]} .
\end{aligned}
$$

The reduced coordinate $u$ is defined as $u=a^{2} / 2 \lambda z$, where $a$ is the pupil radius, $\lambda$ is the wavelength, and $z$ is the axial distance from the zone plate (ZP). The parameter $N$ gives the number of segments forming the fractal structure, and $S$ is the fractal level. In this work we consider only the case where $N=2$.

A similar expression ${ }^{5}$ can be obtained for a conventional FZP:

$$
I_{\mathrm{ZP}}(u, N, S)=\frac{\sin ^{2}\left[\frac{2 \pi M u}{(2 N-1)^{S}}\right]}{\cos ^{2}\left[\frac{\pi u}{(2 N-1)^{S}}\right]} .
$$

Here $M$ is the number of transparent Fresnel zones and is given by the integer $[(2 N-1) S] / 2$.

For both the FZP and the ZP we find major foci at values of $z=f, f / 3, f / 5$, etc., where $f$ is the focal length of the ZP. For this case we define the reduced focal coordinate as $u_{0}=a^{2} / 2 \lambda f$. Equations (1) and (2) show that the major focus at $f$ occurs at values of $u_{0}$ that increase with the choice of parameter $S$ as $u_{0}=4.5,13.5$, and 40.5 for values of $S=2,3$, and 4, respectively. Figure 1 shows a plot of Eq. (1) for $N=2$ and $S=2$ as a function of $z$, using our designed FZP. One can see the major focus and a number of subsidiary focal points surrounding the focus region at $z=1700 \mathrm{~mm}$.

However, the fabrication technique in Ref. 4 requires a constant value of radius $a$ as $S$ increases. Consequently, when we increase the value of $S$, the focal length will decrease as $u_{0}$ increases. Therefore experimental comparison is difficult because the diameter of the focused beam and the focal length change as $S$ varies. Instead, we introduce a different approach to generating these lenses that retains the same focal length for all values of $S$. Accordingly, the radius of lens $a$ must increase as $S$ increases. 


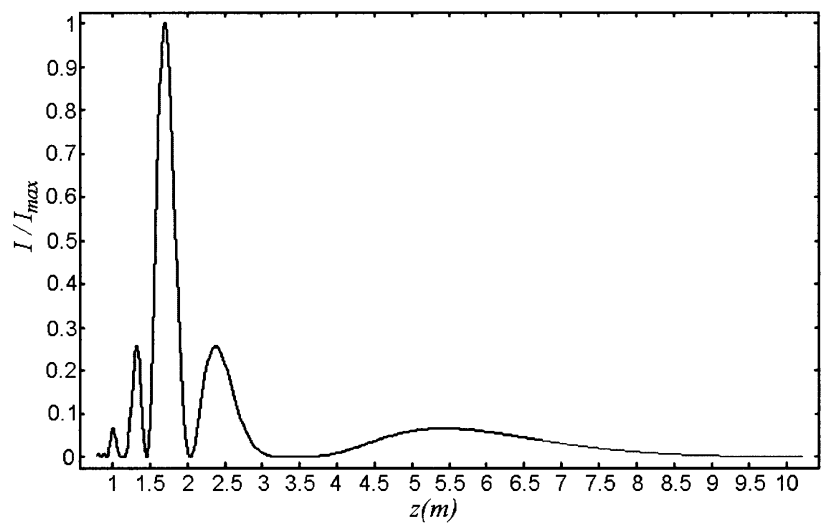

Fig. 1. Intensity as a function of longitudinal coordinate $z$ for a FZP with $N=2$ and $S=2$. For simplicity we display a region up to $z=6 f$.
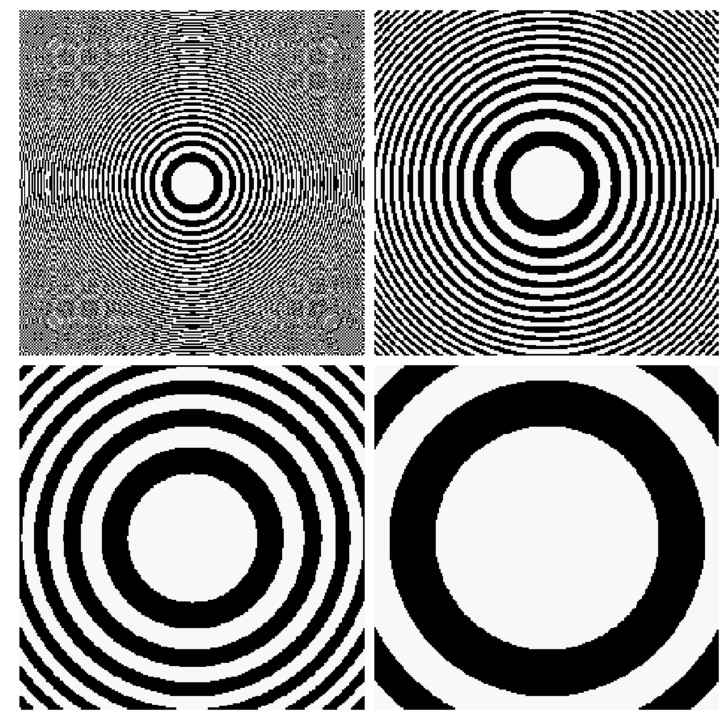

Fig. 2. Ordinary FZPs with focal distances $f$ (top left), $3 f$ (top right), $9 f$ (bottom left), and $27 f$ (bottom right). These plates are used for experimental implementation of FZPs in a LCD.

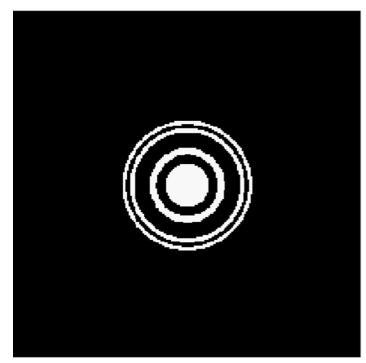

(a)

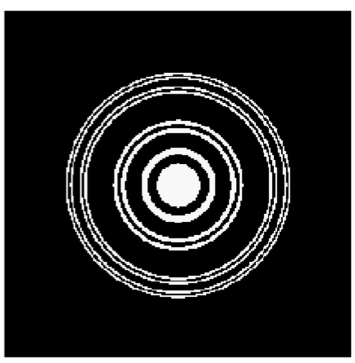

(b)
Fig. 3. FZPs with (a) $N=2$ and $S=2$ and (b) $N=2$ and $S=3$. See the text for details on the theoretical formulation for the intensity transmittance.

Our approach to designing these FZPs is summarized in Figs. 2 and 3 . Figure 2 shows a series of binary Fresnel lenses with $512 \times 512$ pixels and focal lengths of $f, 3 f, 9 f$, and $27 f$, where $f=1700 \mathrm{~mm}$ in our experimental setup. These lenses are encoded with the white areas as +1 and the dark areas as 0 .
To form the $S=2$ lens we multiply the $f$ lens by the $3 f$ lens. We then use an aperture defined by the central dark spot of the $9 f$ lens. Radius $a$ is 96 pixels, or $3.1 \mathrm{~mm}$, with our SLM. The resulting lens is shown in Fig. 3(a). Similarly, to form the $S=3$ lens, we multiply the $f, 3 f$, and $9 f$ lenses and then multiply by the aperture defined by the $27 f$ lens. In this case radius $a$ is 166 pixels, or $5.3 \mathrm{~mm}$, with our SLM. The resulting lens has the same focal length and is shown in Fig. 3(b). These lenses are entirely equivalent to those in Ref. 4, except that they have the same focal lengths.

For the experimental implementation we used a Hitachi Model MTM25v01 twisted nematic LCD with $640 \times 480$ pixels and a pixel spacing of $32 \mu \mathrm{m}$ and operated it in a binary phase-only mode, using previously reported techniques with which the polarization eigenvectors for the display

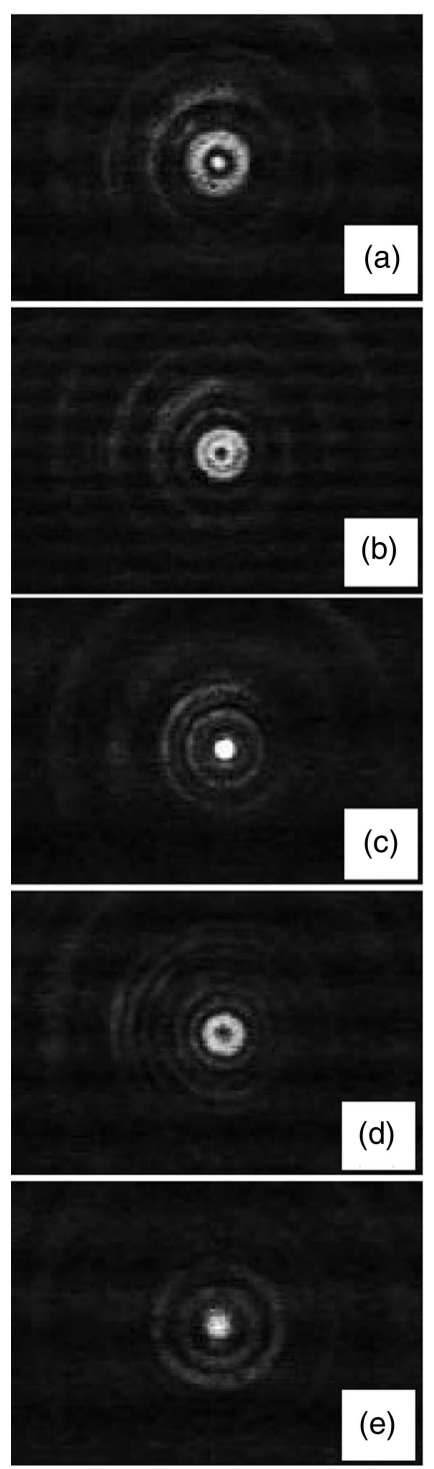

Fig. 4. Series of five focal regions obtained with the FZP as in Fig. 3(a) implemented in a LCD display: (a) $z=$ $1335 \mathrm{~mm}$ (with a precision of $\pm 2 \mathrm{~cm}$ ), (b) $z=1445 \mathrm{~mm}$, (c) $z=1700 \mathrm{~mm}$, (d) $z=2050 \mathrm{~mm}$, (e) $z=2370 \mathrm{~mm}$ (with a precision of $\pm 2.5 \mathrm{~cm}$ ). 
were generated and detected. ${ }^{6-8}$ The LCD was illuminated with collimated light from a $\mathrm{He}-\mathrm{Ne}$ laser and focused by the FZP encoded onto the LCD. The focused spots were detected with a CCD camera as a function of distance from the LCD.

Figure 4 shows recorded intensities at different distances for the $S=2$ fractal lens. We find good agreement. One can see focused spots at the predicted distances of $1335 \pm 20 \mathrm{~mm}, 1700 \pm 25 \mathrm{~mm}$, and $2370 \pm 25 \mathrm{~mm}$ in Figs. 4(a), 4(c), and 4(e), respectively. Note that the diameters of the focused spots increase as the focus length increases, as expected. In addition, the primary focus at $1700 \mathrm{~m}$ is more intense than the other focused spots, as predicted in Fig. 1. At the positions of $1445 \mathrm{~nm}$ in Fig. 4(b) and $2050 \mathrm{~mm}$ in Fig. 4(d), we find the predicted minima characterized by a concentric doughnut form. Another focus at approximately $z=3 f$ is also observed, and another minimum is observed at approximately $z=2 f$, as predicted in Fig. 1. These are not shown. Similar results were found for the $S=3$ case, where the number of focus spots increases as predicted by theory. These are not shown for brevity.

Finally, we were able to increase the value of radius $a$ to the full 256 pixels of the SLM while retaining the order $S=2$. This was accomplished by multiplying the $f$ lens by the $3 f$ lens but without multiplying by the aperture from the $9 f$ lens. In this case, we see a much larger number of focal points that are not predicted by the theory in Ref. 4. We are analyzing a general theoretical approach and plan on presenting this analysis in the near future.
In conclusion, we have shown what are believed to be the first experimental results for fractal zone plates. We introduce an alternative method of generating triadic Cantor FZPs that retains the focal distance while increasing the fractal order $S$. We generally find agreement with the theory. However, the increase in the number of focus spots as we increase parameter $a$ needs additional study.

This research was partially supported by project TIC2002-1846, Ministerio de Ciencia y Tecnologia, Spain. J. A. Davis's e-mail address is jdavis@sciences. sdsu.edu.

\section{References}

1. B. B. Mandelbrot, The Fractal Geometry of Nature (Freeman, San Francisco, Calif., 1982).

2. J. Uozumi and T. Asakura, in Current Trends in Optics, J. C. Dainty, ed. (Academic, Cambridge, 1994), pp. 83-94.

3. D. Rodriguez Merlo, J. A. Rodrigo Martín-Romo, T. Alieva, and M. L. Calvo, Opt. Spectrosc. 95, 131 (2003).

4. G. Saavedra, W. D. Furlan, and J. A. Monsoriu, Opt. Lett. 28, 971 (2003).

5. A. Boivin, J. Opt. Soc. Am. 42, 60 (1952).

6. J. A. Davis, I. Moreno, and P. Tsai, Appl. Opt. 37, 937 (1998).

7. I. Moreno and J. A. Davis, Opt. Eng. 37, 3048 (1998).

8. J. A. Davis, K. O. Valadez, and D. M. Cottrell, Appl. Opt. 42, 2003 (2003). 